\title{
Juvenile Psammomatoid Ossifying Fibroma of Fronto-Ethmoid Complex mimicking Fibrous Dysplasia: Case Report
}

\author{
Adhikari K, Pant AR, Koirala S \\ Department of Radiodiagnosis and Imaging, BPKIHS, Dharan, Nepal
}

Received: March 20, 2018

Accepted: April 30, 2018

Published: June 30, 2018

Cite this paper:

Adhikari K, Pant AR, Koirala S. Juvenile Psammomatoid Ossifying Fibroma of Fronto-Ethmoid Complex mimicking Fibrous Dysplasia: Case Report. Nepalese Journal of Radiology 2018;8(11):33-36.http://dx.doi. org/10.3126/njr.v8i1.20454

\begin{abstract}
Juvenile Psammomatoid Ossifying Fibromais a rare fibro-osseous tumor seen in children and adolescentand mostly arising from the cranio-facial bone. We report a case of 18-year-old boy who presented with diplopiaand progressive right fronto-orbital swelling. On plain radiograph and CT, it was diagnosed as fibrous dysplasiaand mucocele as differential diagnosis. The tumor was resected and histopathological examination showed psammomatoid features. Therefore, the diagnosis of Juvenile Psammomatoid Ossifying Fibroma must be based on both radiological and histopathological findings.
\end{abstract}

Keywords: Diplopia; Ethmoid Sinus; Fibroma, Ossifying

\section{INTRODUCTION}

Juvenile ossifying fibroma (JOF) is a benign fibro-osseous tumor commonly seen in young patient in cranio-facial region. It is classified under variant of ossifying fibroma. ${ }^{1}$ There are two known histological variants of JOF: Juvenile Psammomatoid Ossifying Fibroma (JPOF) and Juvenile Trabecular Ossifying Fibroma (JTOF). ${ }^{2}$ JPOF is locally aggressive tumor and can be confused for malignant lesion. It is very difficult to diagnose JPOF by only looking at the radiological findings as they may resemble other fibrous osseous lesions in similar locations. Here we present a case of JPOF involving the fronto-ethmoid complex mimicking Fibrous Dysplasia (FD).

Correspondence to: Dr. Kapil Adhikari

Department of Radiodiagnosis and Imaging BPKIHS, Dharan, Nepal

Email: adhkapil123@gmail.com 


\section{CASE REPORT}

A18-year-old male presented to the OPD with history of diplopia and swelling on superior aspect of right eye for few months. On physical examination, patient hadproptosis of the right eye. His medical and family histories were unremarkable. Plain radiographof skull bone followed by contrast enhanced computed tomography (CT) of the brain and orbit was done. Plain radiograph showed an expansile lytic lesion of size $5 \times 4 \mathrm{~cm}$ involving right frontal and ethmoidal sinuses (Fig 1).

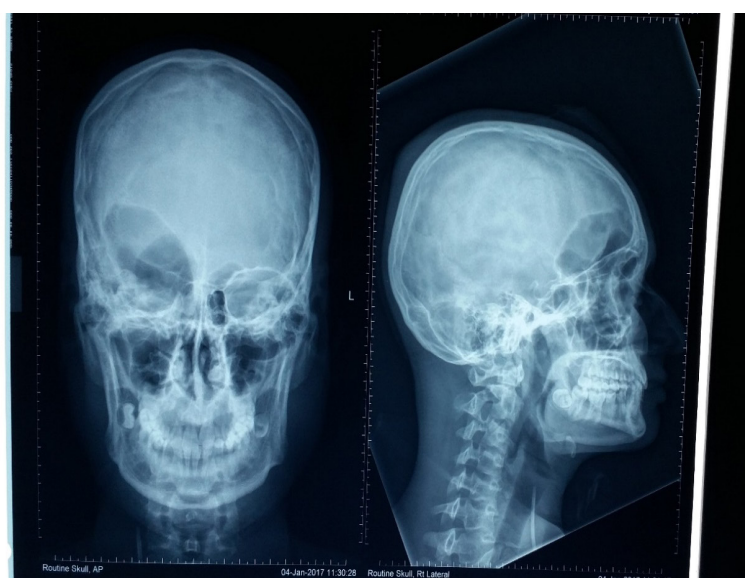

Figure 1: Xray Skull AP and lateral view showing expansile lytic lesion involving right frontoethmoid complex

CT scan showed a well-defined expansile lytic lesion predominately involving the superior wall of the orbit causing compression of the orbital cavity and thinning out of the inner table of right frontal bone. The lesion was predominantly of fluid attenuation with few areas of ground glass type of matrix in the lateral aspect (Fig 2). No significant enhancement was noted on post contrast study. On the basis of clinical scenario and radiological features of benign nature and few areas of ground glass matrix within, we considered fibrous dysplasiaas possible diagnosis with mucocele of frontal sinus as close differential diagnosis. The patient underwentright-sided frontal craniotomy and total excision of the tumor was done and defect was covered with bone graft. The excision material was submitted as right frontal sinus mucocele with bone fragments. On gross examination, multiple bits of irregular greywhite to grey brown to blackish soft tissue was observed. Cut section showed solid grey white appearance. Representative sections examined revealed histological features consistent with benign fibro osseous lesion-Juvenile Psammomatoid Ossifying Fibroma.

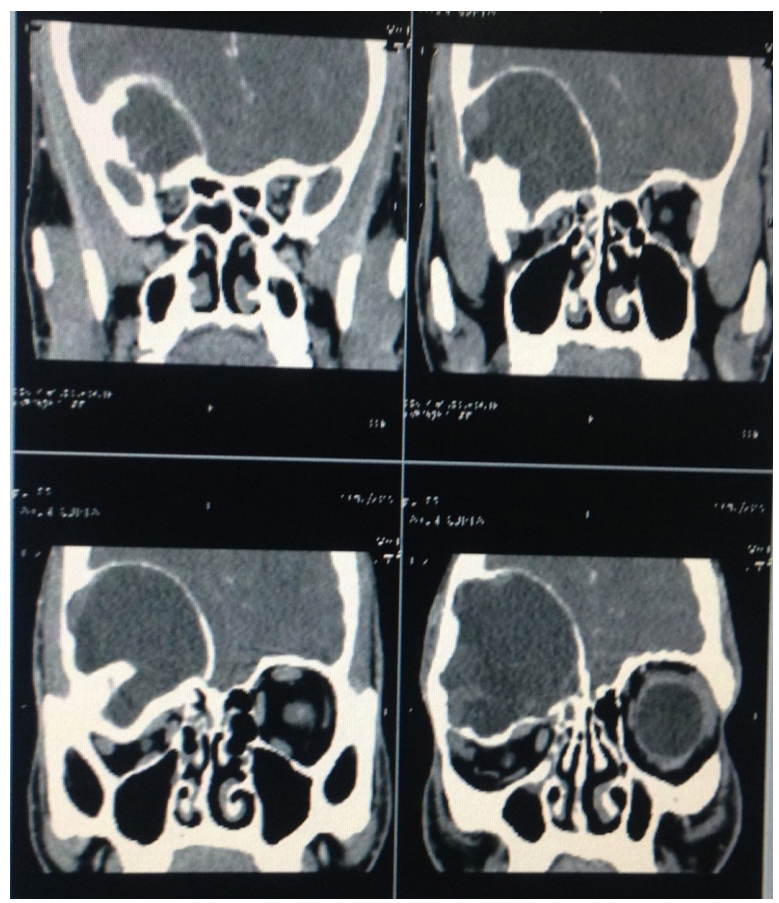

Figure 2: CT scan orbit and PNS (coronal section) of a case of JPOF. The tumor appears as a well-defined expansile lesion predominately involving the superior wall of the orbit with thinning out of the inner table of right frontal bone. The lesion appears predominantly of fluid attenuation with few areas of ground glass type of matrix in the lateral aspect

\section{DISCUSSION}

Ossifying fibroma are categorized as conventional and juvenile forms. ${ }^{2}$ The psammomatoid type of Juvenile Ossifying Fibroma is more common than the trabecular 
variety. It is also moreaggressive and has a high tendency to recur compared to trabecular variety. JPOF is a relatively rare fibro-osseous lesion occurring in children and youngadults. In our case, the patient was in his late teen. JPOF usually occurs in orbit and paranasal sinuses as seen in our case while JTOF usually occurs in maxilla and mandible. ${ }^{3,4}$ Among facial bones, the paranasal sinuses are affected in $90 \%$ of JPOF cases among which the ethmoid sinus is the most common site. ${ }^{3,5,6}$ In our case, the tumor was affecting both frontal and ethmoidal sinuses. The most common complaint in patient with JPOF is proptosis. $^{5}$ Other uncommoncomplaints include swelling of the face, disturbance in ocular movement, headache, and sinusitis, depending on the location of the tumor. ${ }^{5}$ In our case, the patient presented with facial swelling along with proptosis of right eye.

On conventional $\mathrm{X}$ ray, JPOF appears as round oval expansilelytic lesion with welldefined margin. Similar findings were noted in our study. The radiographic appearance can varyranging fromcystic to sclerotic depending upon the tumor content. ${ }^{3}$

On CT scan, JPOF is seen as enhancing soft tissuetumor along with multiple internal foci of calcifications and areas of fluid attenuation within due to cystic changes. These cystic changes may appear multiloculateddue to thin internal septations. ${ }^{6,7}$ Some of the cystic areas may show hemorrhage within and subsequently develop secondary ABCs. JPOF mostly has well-defined margin, although some cases may show disruption of the surrounding sclerotic rim. ${ }^{8}$ Multiple bones may be involved along with bowing and thinning of the involved bones. JPOF may show areas of "ground glass" matrixwithin the lytic area as seen in our case mimicking the appearance of fibrous dysplasia.

The most important differential diagnosis of
JPOF is fibrous dysplasia. ${ }^{6}$ JPOF is mostly unilocular, monostotic and has oval shape with well-defined marginand oftenlacks typical ground-glass attenuation along with multiple punctate areas of calcification within. ${ }^{6,7}$ Similarly, FD tends to be more elongated rather than ovoid appearing JPOF and the border of fibrous dysplasia is ill-defined and converges with surrounding normal bone. ${ }^{9} \mathrm{FD}$ often appears as poorlycircumscribed lesion causing bony expansion and thinning of outer cortex and shows variable radiolucent areas within depending on the proportion of fibrous components giving a characteristic "ground glass" appearance on CT scanning. ${ }^{10}$

Other differential diagnosis of JPOF includemucocele, ABC, osteoblastoma, osteosarcoma and cemento-osseous dysplasia and cementifying fibromas. ${ }^{3,5}$ Mucocele may be associated finding with ossifying fibroma thereby causing difficulty in diagnosis. ${ }^{11}$

JPOF is a rare clinical entity often misdiagnosed because of its rapidly growing and aggressive nature. The present case was also misdiagnosed as fibrous dysplasia. The distinction between psammomatoid variant of juvenile ossifying fibroma, when it affects the paranasal sinuses and fibrous dysplasia, can be quite challenging especially when both present with ground glass opacity. Ossifying fibroma and fibrous dysplasia may share similar radiological features. Hence, the pathological findings of the lesion should also be evaluated side by side when radiological differentiation is uncertain.

\section{CONCLUSION}

Careful assessment of clinical, radiographic and histopathologic features is necessary fordifferentiationof JPOF from other fibroosseous lesions and to arrive at the appropriate diagnosisand fornecessary therapeutic intervention.

CONFLICT OF INTEREST 
None

\section{SOURCES OF FUNDING}

None

\section{REFERENCES}

1. Barnes L, Eveson JW, ReichartP, Sidransky D. WorldHealthOrganizationclassification of tumours. Pathology and genetics of head and neck tumours. International Agency of Research on Cancer 2005. Available from: https://www. iarc.fr/en/publications/pdfsonline/pat-gen/bb9/B B 9.pdf [Accessed 20th Jan 2018].

2. Slootweg PJ, Eveson JW. Tumours of the oral cavity and oropharynx. In World Health Organization Classification of Tumours Pathology \& Genetics Head and Neck Tumous IARC Press, Lyon, France 2005:p166-175 Available from: http://www.iarc. fr/en/publications/pdfs-online/ pat-gen/bb9/bb9-chap 4.pdf [Accessed 20th Jan 2018].

3. Johnson LC, Yousefi ME, Vinh TN, Heffner DK, Hyams VJ, Hartman KS. Juvenile active ossifying fibroma. Its nature, dynamics and origin. Actaotolaryngol Suppl 1991;488:1-40. Available from: https://www.ncbi. nlm.nih.gov/pubmed/1843064 [Accessed 21th Jan 2018].

4. Makek M. Clinical pathology and differential diagnosis of fibro-osseous lesions of the maxillofacial areanew aspects. Dtsch Z Mund Kiefer Gesichtschir 1986;10(3):212-217. https://www.ncbi.nlm.nih.gov/ pubmed/3482011

5. Margo CE, Ragsdale BD, Perman KI, Zimmerman LE, Sweet DE. Psammomatoid (juvenile) ossifying fibroma of the orbit. Ophthalmology 1985;92(1):150-159. https://doi
org/10.1016/S0161-6420(85)34070-8

6. Chung EM, Smirniotopoulos JG, Specht CS, Schroeder JW, Cube R. Pediatric orbit tumors and tumorlike lesions: nonosseous lesions of the extraocular orbit. Radiographics 2007;27(6):1777-1799. https://doi.org/10.1148/rg.276075138

7. Bendet E, Bakon M, Tadmor R, Talmi YP, Kronenberg J. Juvenile cemento-ossifying fibroma of the maxilla. Ann. Otol. Rhinol. Laryngol 1997;106(1):75-78.

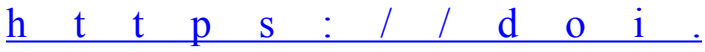
org/10.1177/000348949710600113

8. Margo CE, Ragsdale BD, Perman KI, ZimmermanLE,SweetDE.Psammomatoid (juvenile) ossifying fibroma of the orbit. Ophthalmology 1985;92(1):150-159. https://doi.org/10.1016/S0161$\underline{6420(85) 34070-8}$

9. Morris MR, Blakeslee DB, Zajtchuk JT. Aggressive paranasal sinus ossifying fibroma. Ear Nose Throat J 1989;68(3):260-264. Available from: https://www.ncbi.nlm. nih.gov/pubmed/2663431[Accessed 20th Jan 2018].

10. Wenig BM, Vinh TN, Smirniotopoulos JG, Fowler CB, Houston GD, Heffner DK. Aggressive psammomatoid ossifying fibromas of the sinonasal region. A clinicopathologic study of a distinct group of fibro-osseous lesions. Cancer 1995;76(7):1155-1165. https://doi.org/10.1002/10970142(19951001)76:7<1155::AIDCNCR2820760710>3.0.CO;2-P

11. Kendi AT, Kara S, Altinok D, Keskil S. Sinonasal ossifying fibroma with fluidfluid levels on MR images. AJNR Am $J$ Neuroradiol 2003;24(8):1639-1641. Available from: https://pdfs. semanticscholar.org/1 b $74 /$ e702aaff59e7f28c0d805a6762c3fe36361b. pdf [Accessed 20th Jan 2018]. 